\title{
The Fractionation of Potato Starch
}

\author{
By R. H. HOPKINS AND B. JELINEK (Prague) \\ Department of Brewing and Industrial Fermentation, University of Birmingham
}

(Received 24 July 1947)

The fractionation of starch by precipitation with butanol and similar alcohols, first developed by Schoch (1942), stimulated research in this field by providing the first efficient method of preparing in reasonably pure form the components, amylose and amylopectin. These names have often been applied to the corresponding fractions obtained by earlier methods in various degrees of purity, and in consequence are associated in the literature with many conflicting statements. They are nevertheless retained here, it being understood that amylose, consisting essentially, if not solely, of unbranched chains of $\alpha$-glucopyranose units, is the fraction precipitated from starch paste by butanol, amylopectin remaining in dispersion.

It is supposed that amylose is completely hydrolyzed to maltose by $\beta$-amylase, although the evidence for this is not entirely satisfactory, since pure substrate and enzyme have rarely, if ever, been employed in the same experiment. Tests of the purity of starch fractions include the limit of $\beta$-amylase action, analysis of iodine coloration and potentiometric titration with iodine; the first two have been extensively employed in this work. In the present investigation, attempts have been made to obtain pure amylose and amylopectin by the known precipitation methods, and if possible to improve upon these methods.

Schoch (1942) autoclaved starch paste with butanol or isoamyl alcohol, and later (Schoch, 1945), after autoclaving alone, added excess of pentasol, a commercial mixture of primary amyl alcohols. The liquid was cooled very slowly, amylose coming out in crystalline needles or rosettes which were separated by high-speed centrifugation. It was purified by recrystallization from the same solvent mixture. Amylopectin was precipitated from the mother liquor with methanol. Autoclaving is considered by Schoch (1945) to be essential to the success of the fractionation; otherwise, the fractions are markedly contaminated with one another.

Haworth, Peat \& Sagrott (1946) precipitated the amylose from non-autoclaved starch paste in the cold with thymol. The avoidance of autoclaving is a valuable feature of this method, since mild hydrolytic decomposition of amylose and amylopectin, even in $2 \mathrm{hr}$. at $120^{\circ}$ at $\mathrm{pH} 6$ as employed by Schoch, may be feared. These authors also suggested the use of the cyclohexanols as precipitants, and Whistler \& Hilbert (1945) that of the nitroparaffins.

\section{EXPERIMENTAL}

Materials. Commercial samples of potato starch, the best obtainable, were employed. One sample gave a paste of $\mathrm{pH} .4 .5$. By washing the starch three times with $0.001 \mathrm{~N}$ $\mathrm{K}_{2} \mathrm{CO}_{3}$ followed by distilled water, the reaction of the paste was changed to $\mathrm{pH}$ 5.8-6 as required by Schoch (1942). This starch yielded about $17 \%$ of purified amylose. Another sample, for example, yielded $20 \%$ amylose and a less viscous starch paste.

Starch was mixed to a cream with cold water, and added to boiling water with vigorous stirring. After gentle boiling for $30 \mathrm{~min}$. to yield a homogeneous paste, the latter was either (1) treated with $0.05 \% \mathrm{NaCl}$ and rapidly cooled, or (2) heated in an autoclave at 18-20 lb. pressure for $2 \mathrm{hr}$. as described by Schoch, the $\mathrm{NaCl}$ being omitted. The pastes contained about $1.5 \%$ of starch.

Determinations of yields of fractions. A weighed sample of a solution was evaporated to dryness, dried at $104^{\circ}$ and allowance made for any non-carbohydrate constituent if present.

Determination of blue value. The procedure of McCready \& Hassid (1943) was followed with slight modification, but different units were employed. A solution containing $1 \mathrm{mg}$. of carbohydrate $/ 100 \mathrm{ml}$. and $0.002 \%$ of $\mathrm{I}_{2}$ in $0.02 \% \mathrm{KI}$ was read in a $\mathbf{l ~ c m}$. cell in a Spekker absorptiometer against iodine, using (unless otherwise stated) the 608 filter (6500$7000 \mathrm{~A}$. transmission), which corresponds closely to that employed by McCready \& Hassid. The reading under these conditions is the blue value. In some investigations, other filters, e.g. 607 (5900-6300 A.), were also employed (see text).

\section{Fractionation procedure}

Preliminary experiments yielded $23 \%$ of crude and $17 \%$ of fully purified amylose from one potato starch by Schoch's (1942) method, using butanol. Purification was achieved by redissolving the crude amylose to $0 \cdot 7-1 \cdot 0 \%$ concentration in hot water saturated with butanol, and cooling from $80^{\circ}$ or $90^{\circ}$ over a period of 3-5 hr. The precipitate was washed with butanol-saturated water until the washings no longer gave any colour reaction with $\mathrm{I}_{2}$, redissolved at $80^{\circ}$, reprecipitated and washed. Three reprecipitations, involving nine washings, yielded a product from which no more amylopectin could be extracted, as indicated by the absence of iodine coloration or dissolved carbohydrate in the mother liquor.

Amylose precipitated by thymol (Haworth et al. 1946), or cyclohexanol followed by thymol, was separated as soon as possible by centrifuging, redissolved in hot butanol-saturated water, reprecipitated and washed several times as described above. 
The original mother liquor was concentrated in vacuo and the amylopectin precipitated with ethanol. Foaming created difficulties when thymol had been used, and the evaporation was sometimes omitted.

The best amylopectin was obtained as follows : To 1.5-2\% starch paste (prepared with $\mathrm{NaCl}$ as in (1) above) cyclohexanol was added, drop by drop with stirring, up to 0.15$0.2 \%$ of the paste. Stirring must not be continued until the paste becomes milky. After standing 3 days, $0 \cdot 15 \%$ of powdered thymol was brought into suspension by gentle stirring. The amylose precipitated within 2 days and was separated by centrifuging. The amylopectin had a blue value of about 0.02 , whereas that from the thymol precipitation alone was about $0 \cdot 025-0.03$. If stirring with cyclohexanol increases the opalescence of the paste the resulting amylopectin is found to have an increased blue value.

Precipitation of amylose from starch paste by the method of Haworth et al. (1946), followed by rapid drying, gave a product which was only slightly soluble in water. The original centrifuged preparation could, however, be purified by recrystallization from butanol-saturated water, which was preferred to thymol water for this purpose. In effect, it was thereby converted into the more stable butanol complex, and it was usually stored in this form without drying.

There appears to be no necessity to heat the starch paste under pressure before precipitating the amylose, even if butanol is used. Two preparations of amylose were carried out by butanol precipitation, the one from paste autoclaved for $2 \mathrm{hr}$. at $120^{\circ}$, the other from paste which never rose above $90^{\circ}$ at any time. The latter gave some trouble, but the redissolved amylose was successfully separated from remnants of burst starch granules, which could not be easily removed from the original paste. The two amylose preparations were readily soluble, and gave similar blue values $(0 \cdot 33)$, but that from autoclaved paste was hydrolyzed by $\beta$-amylase much the faster, each reaching the same limit. The corresponding amylopectins possessed identical blue values (0.024), and hydrolyzed at almost the same rates, the $120^{\circ}$ preparation only slightly the faster.

So far as the amylose was concerned it did not greatly matter which precipitant was used, butanol, thymol or cyclohexanol followed by thymol, provided that the separated complex was promptly washed and recrystallized from butanol water. In one instance, using the same nonautoclaved starch paste, butanol and thymol precipitations were performed side by side. The butanol amylose, recrystallized three times, gave a $\mathbf{1 7 \cdot 2} \%$ yield, blue value (B.v.) 0.355. Thymol-precipitated amylose, recrystallized three times from butanol water, gave a $17 \cdot 7 \%$ yield, B.v. 0.345. The light absorptions of the respective blue liquids were almost identical from 4300 to $5800 \mathrm{~A}$. However, the thymol preparation gave the lower absorption from 5800 to $7000 \mathrm{~A}$. Under the same conditions, both amyloses hydrolyzed at the same rate with $\beta$-amylase, and reached the limit of $99.5 \%$ of theoretically obtainable maltose.

The crude thymol-amylose was bulky and did not adhere to the walls of the vessel, but after recrystallization from butanol water it occupied only $40 \%$ as much space as before and adhered to the walls. It underwent no further change in these respects during further recrystallizations from butanol water. It was found unsatisfactory to leave it long in contact with thymol water as it lost solubility and affinity for $I_{2}$.

The butanol-amylose complex, on heating with water saturated with butanol, begins to disperse at $65^{\circ}$ to form an opalescent solution which clears at about $73^{\circ}$. On cooling slowly the phenomenon of supercooling may be observed as far down as $35^{\circ}$.

Schoch $(1942,1945)$ stipulated that the reaction of potato starch paste must be about $\mathrm{pH} 5 \cdot 9-6 \cdot 3$ for successful fractionation. In this work, there were indications that wider variations in $\mathrm{pH}$ were permissible; indeed, successful precipitations from non-autoclaved paste were effected even at $\mathrm{pH} 4 \cdot 1$ and 9 . But certain differences in behaviour were noted in extreme cases. Thus, amylose precipitated from non-autoclaved paste by butanol, thymol or cyclohexanol followed by thymol, brought by $\mathrm{HCl}$ addition to $\mathrm{pH} \mathrm{4} \cdot 1$, was more easily washed and freed from amylopectin than in parallel precipitations at $\mathrm{pH} 6$ and 9 . It also showed a greater tendency to retrograde in neutral, hot aqueous solution, and when dried on a watch glass it was opaque. It was hydrolyzed by $\beta$-amylase in dilute solution faster than amylose precipitated at $\mathrm{pH} 6$ or 9 hydrolyzed under identical conditions.

No significant differences were noted in the amylopectins except that the lowest blue values were obtained in the cyclohexanol + thymol method (Table 2).

\section{RESULTS}

\section{General properties of amylose}

Amylose forms a viscous solution when concentrated, which may cool to a gelatinous consistency. Solutions of over $0.5 \%$ retrograde fairly readily, and this has been a source of difficulty in previous work. Whenever amylose approaching a high degree of purity has been prepared in these laboratories, e.g. by electrophoresis, retrogradation has been liable to occur, rendering the product permanently insoluble in water and useless for many purposes. Precipitates from hot solutions on cooling may not be retrograded material, but may redissolve with more hot water. Retrogradation may be avoided by high temperature, low concentration, maintenance of neutral pH, and over long periods by preservation in the form of a complex with butanol, thymol and nitroparaffins. Recrystallization from butanol water need not involve a cooling period greater than 3-5 hr. In the form of the butanol complex, i.e. in the presence of butanol-saturated water, even the purest amylose is stable for months and shows no signs of retrogradation. The supernatant liquid in such a case contains less than $1 \mathrm{mg}$. solid matter $/ 100 \mathrm{ml}$. and gives only a yellow colour with iodine.

\section{Criteria of purity of amylose}

The distinction between the iodine colorations of amylose and amylopectin consists essentially in the intensity of the blue. While amylopectin shows no great variation from 5000 to 8000 A., amylose exhibits a marked minimum transmission near $6000 \mathrm{~A}$. Kerr (1944) places this at 6000-6200 A. for potato amylose which corresponds to light filter 607 as 
used with the Spekker absorptiometer. For different preparations we have found from 5800 to $6100 \mathrm{~A}$., the highest reading on the absorptiometer being obtained with the 607 filter. After making allowance for variation in experimental error when using these filters, the one should be chosen which gives the greatest numerical difference between amylose and amylopectin. This is when the need is to characterize different preparations of amylose or to measure amylose present es an impurity in amylopectin. For the reverse purpose, that of measuring blue value of both the amylopectin which remains contaminated with it and the amylose which has lost it. A perfectly representative sample of amylose cannot be obtained unless the first precipitation is performed in the right way.

The blue value was found to be unaffected by small quantities of butanol and maltose. It is chiefly influenced by the temperature at the time of reading, and by the conditions under which the amylose has been crystallized from solution, and, generally, the amount of exposure it has had to hot

\section{Table 1. Blue values of amylose after various periods of heating and cooling}

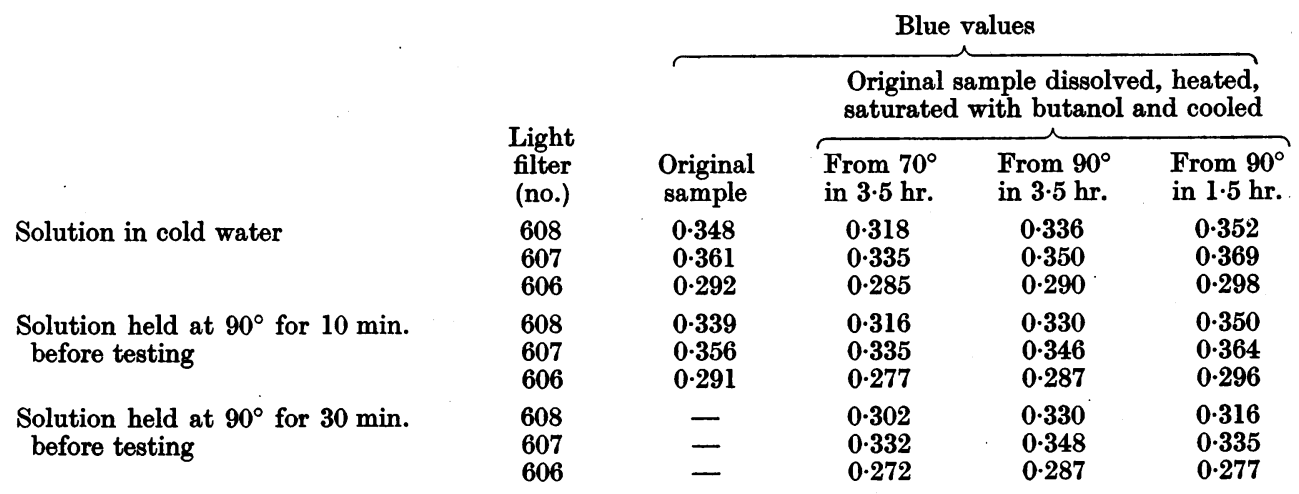

Filter 608, 6500-7000 A.; 607, 5900-6300 A.; 606, 5700-5900 A.

amylopectin or red iodine complexes in amylose, the filter selected should transmit light of about 4800 5000 A., the 603 filter being indicated.

Failure to remove amylopectin or other carbohydrate by recrystallization from cooling butanolwater solution is an indispensable criterion of purity. The process should be accompanied by no change in blue value (so long as recrystallization is brought about under the same conditions and is complete). Short of adequate investigation of molecular structure, perhaps the best further criterion available to us is the completeness of hydrolysis by pure $\beta$-amylase together with some kinetic features of this hydrolysis which are dealt with in a separate communication.

The blue value is of use during the process of freeing amylose from adherent amylopectin, and its value rises to a maximum during successive recrystallizations. But the value may be different in one preparation from that of another and seems to depend both on the initial conditions and on those of the final recrystallization.

Some amylose is normally left behind in the amylopectin, probably relatively low molecular amylose, which is difficult to remove. That fraction of the native amylose which is not precipitated in the first instance will vary from process to process, and from precipitant to precipitant, and will influence the water. Table 1 shows a series of values (measured with three filters) of one specimen dissolved and recrystallized under different conditions. The products (butanol complex) were each dissolved in three ways. These results mainly serve to show that, within the range of the experimental conditions, the slower the cooling the lower the blue value. A smaller influence is exerted by conditions of dissolving, standing at $90^{\circ}$ lowering the value. Evidently, these conditions must be controlled if the blue value is to be used as a criterion.

From this and other experiments it appears that when amylose is unduly exposed to hot water a part may precipitate, this being irreversibly insoluble (retrogradation), whilst more may be changed but remain in solution. The solution now exhibits a lower blue value than before and can no longer be hydrolyzed to $100 \%$ of maltose by $\beta$-amylase. The same is true of amylose which has been precipitated and washed by ethanol and ether. It is less soluble in water and the soluble portion has a lower blue value than before this treatment. This changed, soluble amylose is apparently related to the natural amylose much as denatured protein is to the corresponding native form. On the other hand, retrograded material would seem to correspond to protein which has been both denatured and coagulated. The blue value is not related to the mean chain 
length of the molecules, but to the extent of denaturation. As amylose is purified it shows an increasing tendency to retrograde.

Recrystallization from hot aqueous solution by half saturation with butanol effects no fractionation of amylose so far as is indicated by blue values, provided that two conditions are fulfilled. The precipitated portion and that remaining in solution must each be separately recrystallized from hot butanol-saturated water and redissolved under the same conditions, and there must be no denaturation. If there are any signs of retrogradation, such as marked opalescence, the unprecipitated fraction is found to have a lower blue value than the precipitate, e.g. $0 \cdot 310$ as against $0 \cdot 340$.

\section{Purification and properties of amylopectin}

Amylopectin was not further purified or its blue value lowered by reprecipitation by thymol, butanol, acetone, chloroform, or thymol-cyclohexanol mixture in equal parts, by shaking to facilitate retrogradation of amylose or by filtration through cotton. The latter adsorbed added amylose, however. The blue value was not altered from that of the first amylopectin precipitate, although this value varied markedly according to the original method of precipitating the amylose. Thus, purer amylopectin was obtained by precipitation with thymol than with butanol, and still better with cyclohexanol followed by thymol.

Presumably, the amylose remaining entangled with the amylopectin consists of short molecular chains which the precipitant fails to pick out. Various precipitation procedures were tried. For example, ethanol was added to $1-2 \%$ of amylopectin in $0.05 \% \mathrm{NaCl}$ until a cotton-like precipitate (I) was formed. This was separated and the mother liquor allowed to stand. In the course of the following days a further precipitate slowly formed (ppt. II). The results in Table 2 suggest that a little contaminated amylose was preferentially removed with the first precipitate, but neither preparation is considered to be entirely free from amylose. $\beta$-Amylase indicated no difference between the first and second precipitates, which hydrolyzed at the same rates and reached the same limits.

Amylopectin is in general hygroscopic. It adheres so strongly to glass or porcelain that in the course of drying the shrinking material picks particles of glass from the surface of the vessel. This is specially noticeable on cooling the dried amylopectin, the differences in thermal expansion of glass and amylopectin coming into operation.

\section{SUMMARY}

1. Amylose and amylopectin have been prepared from non-autoclaved potato starch paste by successive additions on alternate days of cyclohexanol and thymol, which, on the whole, gave the best results, and by the methods of Schoch and of Haworth, Peat \& Sagrott.

2. No great differences were noted among the products obtained by use of three precipitants.

3. There is no advantage to be gained by heating the starch paste under pressure. If this is omitted a fairly wide range of pH may be tolerated at the time of precipitation, but the amylose produced under acid conditions showed the greater tendency to retrograde in neutral solution.

4. Preparations of amylose with 17 times the blue value, as measured on the Spekker absorptiometer, of the corresponding amylopectin have been obtained.

5. The blue value of amylose is greatly influenced by the method of recrystallization from butanolsaturated water, and by the period of heating when redissolving.

Table 2. Blue values of amylose and amylopectin

\begin{tabular}{|c|c|c|c|c|c|}
\hline & & & & & \\
\hline - & & & & nylopec & \\
\hline & pH at & & & Ethan & pitates \\
\hline Precipitant & precipitation & Amylose & Crude & $\mathbf{I}$ & II \\
\hline Butanol & $\begin{array}{r}4 \cdot 1 \\
6 \cdot 0 \\
9 \cdot 4\end{array}$ & $\begin{array}{l}0 \cdot 285 \\
0 \cdot 310 \\
0 \cdot 320\end{array}$ & $\underline{0.024}$ & $\begin{array}{c}0.028 \\
\overrightarrow{0.027}\end{array}$ & $\begin{array}{l}0.025 \\
0 . \overline{025}\end{array}$ \\
\hline Thymol & $\begin{array}{l}4 \cdot 1 \\
6 \cdot 0 \\
9 \cdot 4\end{array}$ & $\begin{array}{l}0 \cdot 302 \\
0 \cdot 332 \\
0 \cdot 342\end{array}$ & - & $\begin{array}{c}0.029 \\
\overline{0.028}\end{array}$ & $\begin{array}{l}0.025 \\
\overrightarrow{0.025}\end{array}$ \\
\hline cycloHexanol followed by thymol & $\begin{array}{l}4 \cdot 1 \\
6 \cdot 0 \\
9 \cdot 4\end{array}$ & $\begin{array}{l}0.318 \\
0.336 \\
0.334\end{array}$ & $\begin{array}{c}\overline{0.021} \\
-\end{array}$ & $\begin{array}{c}0.028 \\
\overline{0.027}\end{array}$ & $\begin{array}{c}0.022 \\
\overline{0} \cdot \overline{023}\end{array}$ \\
\hline
\end{tabular}


6. Exposure of amylose to hot water brings about changes even in the portion which does not retrograde. The blue value falls and $\beta$-amylase action is incomplete.

7. Amylopectin was not so successfully purified, but fractional precipitation with ethanol freed it slightly from amylose which had escaped the first precipitation.

The authors are indebted to Mr L. E. Harrison for assistance in experimental work. One of us (B.J.) is indebted to the British Council for financial assistance which enabled this work to be performed.

\title{
REFERENCES
}

Haworth, W. N., Peat, S. \& Sagrott, P. E. (1946). Nature, Lond., 157, 19.

Kerr, R. W. (1944). Chemistry and Industry of Starch. New York: Academic Press.

McCready, R. M. \& Hassid, W. Z. (1943). J. Amer. chem. Soc. 65, 1154.
Schoch, T. J. (1942). J. Amer. chem. Soc. 64, 2957.

Schoch, T. J. (1945). Advances in Carbohydrate Chemistry, 1.

New York: Academic Press.

Whistler, R. L, \& Hilbert, G. E. (1945). J. Amer. chem. Soc. 67, 1161.

\section{The Action of $\beta$-Amylase on Potato Amylose}

\author{
By R. H. HOPKINS, B. JELINEK AND L. E. HARRISON \\ Department of Brewing and Industrial Fermentation, University of Birmingham
}

(Received 24 July 1947)

It has long been known that almost all natural starches contain a constituent which can be hydrolyzed to completion by $\beta$-amylase. It has been given various names, that of amylose being the one used here. It is only in quite recent years that amylose has been prepared in a state of purity (Schoch, 1942; Haworth, Peat \& Sagrott, 1946). $\beta$-Amylase has usually been reported as promoting the hydrolysis of amylose to $90-100 \%$ of maltose, but many of these figures may be above the truth. Often, it has not been made clear whether the figure reported referred to maltose expressed as a percentage of the moisture-free starch used or of the maltose theoretically obtainable from it. The absence of glucose from the products has been assumed, although many preparations of $\beta$-amylase contain maltase. Lastly, it is rare that the $\beta$-amylase has been demonstrated to be free from $\alpha$-amylase and from maltase.

In this work, the purest amylose and $\beta$-amylases obtainable have been investigated with special attention to the kinetics of the reaction and a hypothesis is advanced to account for the facts.

\section{MATERIALS AND METHODS}

Substrates. The various preparations of amylose are described elsewhere (Hopkins \& Jelinek, 1948). Solutions not exceeding $0.9 \%$ concentration were prepared by heating the butanol-amylose complex in water until the butanol was driven off.

Blue value (B.v.). The procedure of McCready \& Hassid (1943) as modified by Hopkins \& Jelinek (1948) was employed.

Red value (R.v.). This was measured in the same way as the B.v., except that filters 602 and 603 were used with the
Spekker absorptiometer. It was found of advantage, however, to increase the iodine concentration from 0.002 to $0.008 \%$.

Enzyme preparations. $\beta$-Amylase was prepared (1) from well-ripened barley as described by Hopkins, Murray \& Lockwood (1946), (2) from soya beans by the method of Newton \& Naylor (1939) and Newton, Hixon \& Naylor (1943). Both specimens were tested and found to be free from maltase activity under the appropriate conditions of use. The limits of hydrolysis of starch pastes were $57 \%$ of the theoretical maltose $\left(R_{m}=57 \%\right)$, and the preparations were considered to be free from $\alpha$-amylase.

Reaction mixtures. These were as employed by Hopkins et al. (1946), but contained varying concentrations of amylose as stated in the tables, and were buffered at $\mathrm{pH} 4.6$ for barley and $\mathrm{pH} \mathrm{5.8}$ for soya amylase. Progress of hydrolysis at $25^{\circ}$ was determined as described by Hopkins et al. (1946), except that the hypoiodite titration was found not to be wholly satisfactory in the presence of certain antiseptics or if a trace of butanol was present. In such cases, the method of Cole (1933) was substituted, the ferricyanide being calibrated against maltose. Withdrawals from the reaction mixture were made into boiling water.

Calculation of the values of ' $k$ ' as if the reaction were monomolecular revealed that these decline progressively and markedly through the reaction. As is shown later, there was no theoretical reason for expecting monomolecularity.

For the determination of the affinity constant, a series of flasks was set up containing varying concentrations of the amylose (about four flasks for each concentration). All flasks contained identical concentrations of enzyme and buffer. After suitable intervals of time (estimated from a pilot experiment) $\mathrm{pH} 10$ buffer was added to the respective flasks to check the reaction and the maltose determined by titration. The velocity of reaction $v(\mathrm{mg}$. maltose $/ 100 \mathrm{ml}$./ min.) was obtained for each concentration of amylose substrate from the initial slope of the progress curves. 